\title{
EFFICIENCY OF ENZYMATIC DEBRIDEMENT IN THE HEALING PROCESS OF CHRONIC WOUNDS IN SMALL ANIMAL PRACTICE
}

\author{
Hamilton, L., Kožár, M. \\ University of Veterinary Medicine and Pharmacy, Komenského 73, 04181 Košice \\ Slovakia
}

martin.kozar@uvlf.sk

\section{ABSTRACT}

Skin wounds are a common presentation in small animal practice. These wounds may be acute or chronic with a complicated healing process. An important aspect of the healing of wounds is debridement which may be carried out by surgical, autolytic, mechanical or enzymatic methods. The debridement method is chosen according to the individual skin defect and influenced by factors such as wound size and location, the age of the wound, and the presence of infection or exudate. Enzymatic debridement is a method that is not commonly used in veterinary practice, and involves the use of enzyme preparations to remove necrotic tissue from a wound. The aim of this study was to investigate the effects of the enzymatic ointment collagenase as a method of debridement, and its effect on the macroscopic appearance of chronic skin wounds in cats and dogs. We observed that the application of Iruxol Mono directly to the wound changes the progress of the healing process, with no obvious adverse effects. The time of healing of chronic wounds was decreased and healthy granulation tissue was developed within a couple of days after application of the ointment. Enzymatic debridement appears to be a promising method of debridement for use in chronic wounds, and should be considered in cases where more conventional methods of debridement are ineffective or unsuitable.

Key words: collagenase; chronic wounds; debridement; enzymes

\section{INTRODUCTION}

Wounds are commonly encountered in small animal practice. In the year 2015, the Small Animal Clinic, section of surgery, orthopaedics, roentgenology and reproduction of the University of Veterinary Medicine and Pharmacy in Košice examined 2172 patients, of which there were 182 cases of wounds. In order to heal successfully, wounds must pass through three phases: inflammation, proliferation and maturation [9]. If this process is interrupted or unsuccessful, a wound may become chronic. Chronic, non-healing wounds require more intervention than acute wounds in 
order to heal successfully. Chronic wounds often contain a large amount of necrotic tissue, decreased blood supply and substantial exudate which impedes healing.

An important aspect in the healing of chronic wounds, and the focus of this study, is debridement. The purpose of debridement is to remove any bacteria and damaged or necrotic tissue in order to improve healing of the remaining tissue [9]. There are four common methods of debridement that may be used alone or in combination. These methods are surgical, autolytic, mechanical and enzymatic. The choice of the method is based on the characteristics of the individual wound such as its size, location, aetiology, age, the presence of infection or exudate, and the overall condition of the patient [2].

Surgical debridement is the most common and rapid method of debridement. It involves the use of a scalpel or other blade to scrape or cut away unhealthy tissue. The disadvantages of surgical debridement are that it can be painful, causes bleeding, and may result in the accidental removal of viable tissue. Autolytic debridement is a slow but safe method that relies on the patient's own healing processes and the presence of phagocytic cells and proteolytic enzymes produced naturally in the wound. These natural processes are enhanced by the application of dressings which maintain a moist wound bed. Mechanical debridement involves removal of unhealthy tissue by force, using methods such as wet-to-dry dressings, lavage or ultrasound. This method may cause further damage and pain to a wounded area, for example the use of wet-to-dry dressings may remove viable tissue along with necrotic tissue.

Enzymatic debridement is not commonly used in veterinary medicine compared to other methods. It involves the direct application of proteolytic enzymes to a wound, which breaks down necrotic tissue. There have been only a few studies on the efficacy of enzymatic debridement in animal patients, however it has been used very successfully in human patients $[5,6]$. Collagenase-based preparations are the most common type of enzymatic debriding agents. Collagenase is a water-soluble proteinase derived from bacteria such as Clostridium histolyticum and Vibrio spp. It has specific activity against collagen in non-viable cells which means it does not break down healthy tissue, making it safe for use in wound management [1]. Studies in human medicine have shown that the use of collagenase increases the speed of healing and reduction in wound size, as well as reducing inflammation $[6,8]$.
There are many advantages of enzymatic debridement over other more commonly used methods. Enzymatic debridement is minimally invasive, and its application can be carried out while the animal is conscious, minimising the necessity for general anaesthesia and its associated risks. Potential disadvantages of enzymatic debridement are that it may not be suitable as the sole treatment in patients with large, deep, burn wounds [4]. It has also been proposed that enzymatic debridement may not be safe in wounds with a high bacterial burden [3]. Payne et al. [7] were able to demonstrate the safety of both collagenase and papain-urea agents in wounds with high bacterial burdens via the use of a rodent model.

Many researchers have attempted to evaluate the efficacy of enzymatic debridement on the healing of wounds in humans, and in animal models under experimental conditions. However, there have been few studies carried out on animal patients. The aim of this study was to assess the healing process of wounds in cats and dogs that have been treated with enzymatic debridement.

\section{MATERIALS AND METHODS}

The enzymatic debriding agent used in this study was Iruxol Mono, a collagenase preparation produced by Smith \&Nephew Ltd. (London, Great Britain). Iruxol contains the enzyme clostridiopeptidase $\mathrm{A}$ and associated proteases, contained in excipients liquid paraffin and white soft paraffin. It is indicated for the debridement of necrotising wounds, such as decubital ulcers in human patients. The recommended method of application is a $2 \mathrm{~mm}$ thickness of ointment once per day, applied directly to the wound or wound dressing.

The animals used in this clinical study were 5 dogs and 2 cats which were patients of the Small Animal Clinic, section of surgery, orthopaedics, roentgenology and reproduction of the University of Veterinary Medicine and Pharmacy in Košice. They were presented with chronic, non-healing wounds during the period between November 2015 and March 2016. Before starting treatment with Iruxol, cultures were taken from the wounds to ensure they were free from bacterial contamination.

The Iruxol ointment was applied once per day directly to the wound and the surrounding skin in a layer of at least $2 \mathrm{~mm}$ thick. The volume of Iruxol used per day in each case 

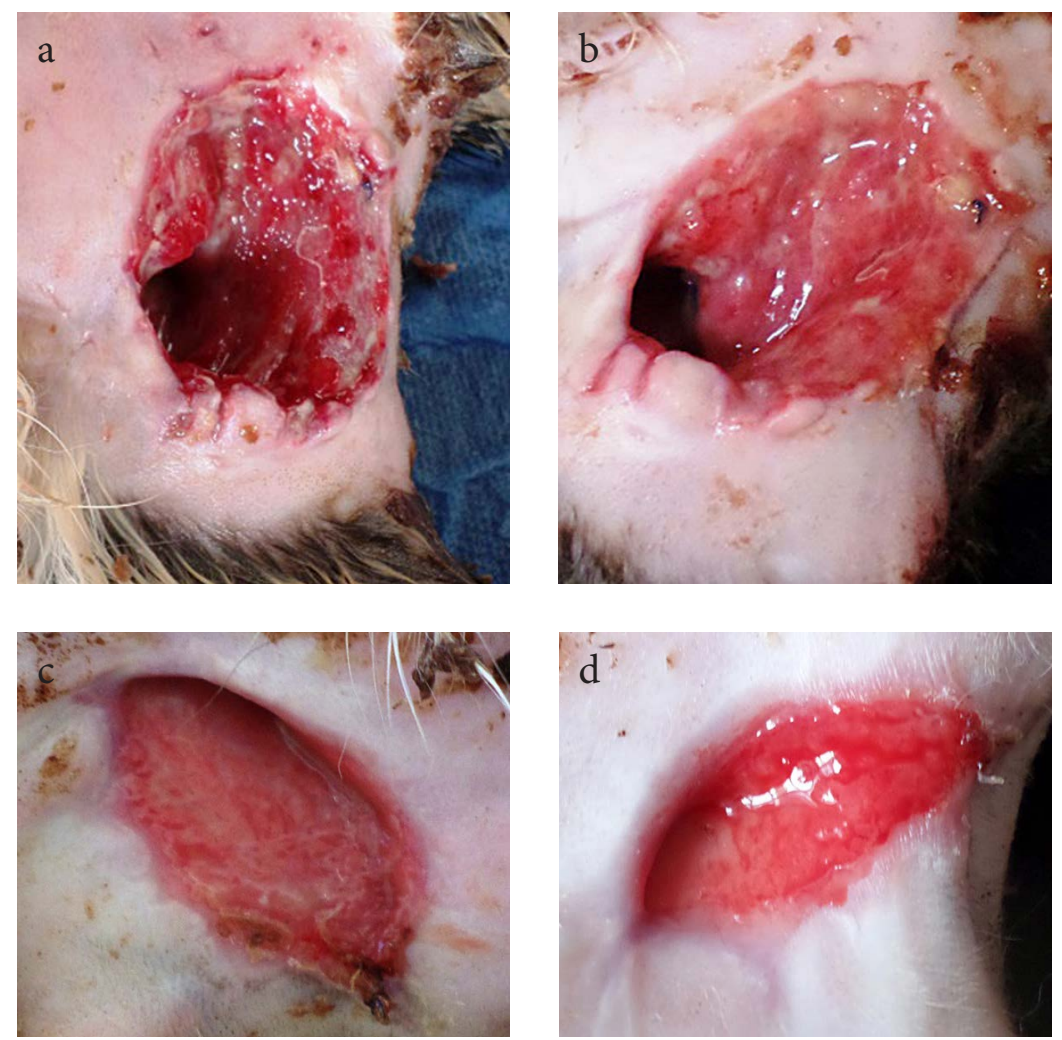

Fig. 1. Wound of the right thigh in a cat

$\mathrm{a}$ - before treatment; $\mathrm{b}$ - after 1 day of treatment; $\mathrm{c}$ - after 8 days of treatment; $\mathrm{d}$ - after 14 days of treatment (Source: Authors)

a
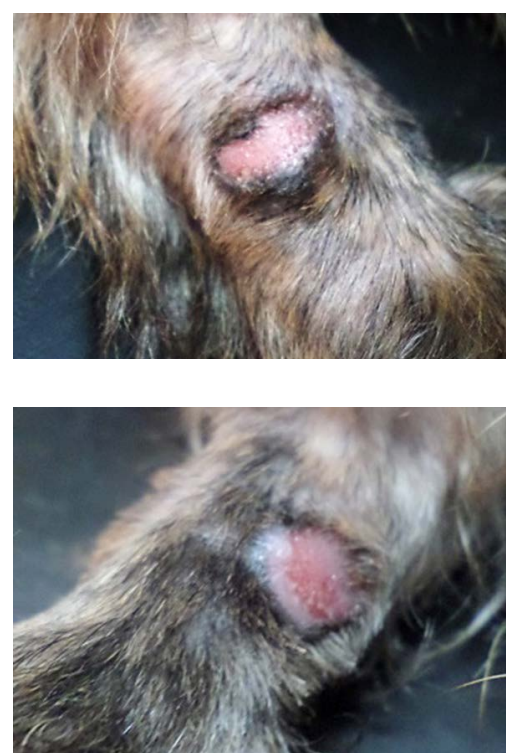

b
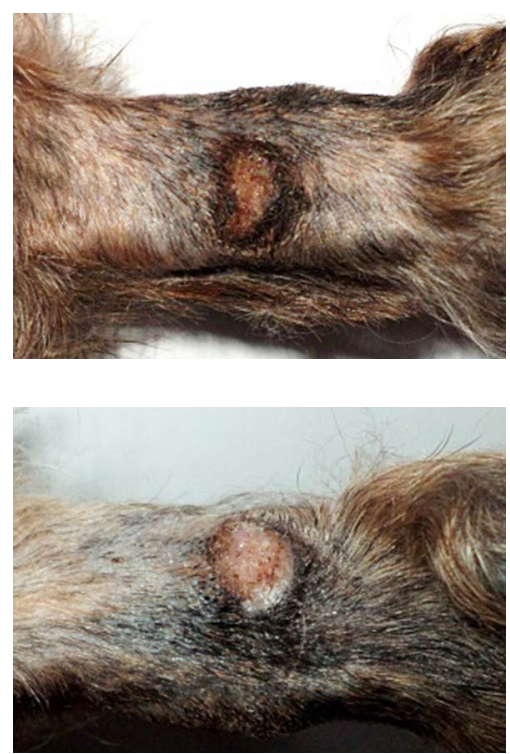
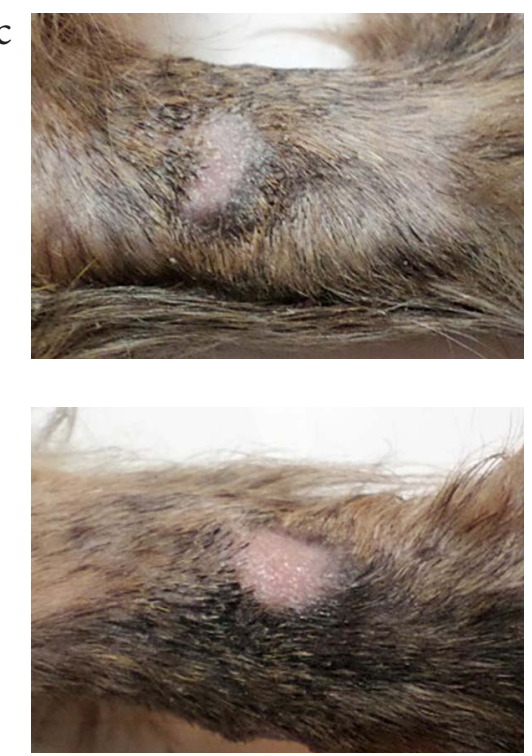

Fig. 2. Wounds of the right tarsus in a $\operatorname{dog}$ (top = lateral side, bottom = medial side) a - before treatment; b - after 1 day of treatment; $\mathrm{c}$ - after 7 days of treatment (Source: Authors) 
was based on the size and depth of the wound. The wound was examined each day to assess the healing process and size of the wound. During the wound healing process, we also examined colour changes, desiccation of wound edges, the production of granulation tissue in the wound bed and production of exudate. Iruxol treatment was stopped when the wound was completely healed, and the time of healing was recorded.

\section{RESULTS AND DISCUSSION}

Enzymatic debridement was carried out in 7 animals; 5 dogs and 2 cats. In 6 out of 7 cases the wounds healed well following enzymatic debridement, even in patients in which other methods of debridement and surgical treatment had previously been unsuccessful. In the remaining patient, the wound reduced in size during the first week of application of Iruxol, however it became contaminated with Staphylococci bacteria and underwent dehiscence so the Iruxol treatment was halted.

In all patients, the production of granulation tissue was observed within 2 days of beginning Iruxol treatment. In a few cases, a small amount of serous wound exudate was observed, which was cleaned from the wound bed before the daily application of Iruxol. The wound edges remained well-defined throughout the treatment and reduction in the wound size progressed rapidly. In some cases, such as the case pictured in Figure 1, there was a visible reduction in the size within 2 days of Iruxol treatment.

The patient in Figure 1 was a kitten which presented with a large, deep wound of unknown aetiology on the medial right thigh. The patient also had desiccated toes on the right hind foot which were amputated during the course of the Iruxol treatment. $0.5 \mathrm{ml}$ of Iruxol was applied directly to the wound once per day and left uncovered, the patient wore an Elizabethan-collar to prevent licking of the wound.

Within 24 hours there was a visual improvement to the surface of the wound. The edges of the wound were cleaner and more defined than prior to the application of Iruxol, and healthy granulation tissue was visible. The wound decreased rapidly in size, beginning on the third day after initiation of Iruxol treatment. Iruxol treatment was continued for 2.5 weeks until the wound was completely healed.

The patient in Figure 2 was a 4 -year old, castrated male mixed-breed dog with a history of atopic dermatitis. The patient had self-inflicted wounds to the lateral and medial right tarsus which had been present for approximately 3 months. $0.2 \mathrm{ml}$ Iruxol was applied once per day to each wound and covered with a bandage to prevent licking.

Within 2 days of beginning Iruxol treatment, the wound reduced in size. Within 1 week, the wound began epithelialization. Iruxol treatment was continued by the owner for approximately 2 weeks until the wounds were completely healed.

In all but one of the cases studied so far, the wounds healed successfully when treatment with Iruxol ointment was used as the sole method of debridement. No negative effects of the treatment were observed. Following treatment with Iruxol, granulation tissue was formed within 2 days, which indicated that the Iruxol treatment improved the growth of granulation tissue.

Studies of enzymatic debridement in human patients have yielded similar results. For example, retrospective evaluations of the use of collagenase for enzymatic debridement in human patients have been published by Marazzi et al [5], and Ramundo and Gray [8]. Both studies found the treatment to be well-tolerated by patients and even to reduce pain at the wound site. Another human study by Onesti et al [6] compared the efficacy of enzymatic and mechanical debridement and showed that cases treated with enzymatic debridement had more rapid reduction in wound size and caused less pain than debridement using wet-to-dry dressings.

Further research into the efficacy of enzymatic debridement in small animal practice should include a comparison with different methods of debridement used in similar wounds, as carried out in human patients by $\mathrm{O}$ nesti et al [6]. Further research should also include histological analysis of the wound before and after enzymatic debridement. However this will be difficult to accomplish in animal patients due to reluctance of the owner and the ethical implications of taking tissue samples for histological analysis.

\section{CONCLUSIONS}

The results observed in this study support the use of enzymatic debriding agents such as Iruxol in the healing process of chronic wounds. The advantages of this method compared to other methods of debridement are that it is non-invasive, painless and can be carried out without an- 
aesthesia. It is therefore worth considering enzymatic debridement for the treatment of chronic wounds in cases where other methods of debridement may be unsuitable.

\section{REFERENCES}

1. Falanga, V., 2002: Wound bed preparation and the role of enzymes: a case for multiple actions of therapeutic agents. Wounds, 14, 47-57.

2. Gokoo, C., 2009: A primer on wound bed preparation. Journal of the American College of Certified Wound Specialists, 1, 35-39.

3. Hummel, R.P., Kautz, P.D., MacMillan, B.G., Altmeier, W. A., 1974: The continuing problem of sepsis following enzymatic debridement of burns. Journal of Trauma, 14, 572-579.

4. Langer, V., Bhandari, P. S., Rajagopalan, S., Mukherjee, M. K., 2013: Enzymatic debridement of large burn wounds with papain-urea: Is it safe? Medical Journal Armed Forces India, $69,144-150$.
5. Marazzi, M., Stefani, A., Chiaratti, A., Ordanini, M. N., Falcone, L., Rapisarda, V., 2006: Effect of enzymatic debridement with collagenase on acute and hard-to-heal wounds. Journal of Wound Care, 15, 222-227.

6. Onesti, M. G., Fioramonti, P., Fino, P., Sorvillo, V., Carella, S., Scuderi, N., 2015: Effect of enzymatic debridement with two different collagenases versus mechanical debridement on chronic hard-to-heal wounds. International Wound Journal, 1742, 1-5.

7. Payne, W. G., Salas, R. E., Ko, F., Naidu, D. K., Donate, G., Wright, T.E., Robson, M.C., 2008: Enzymatic debriding agents are safe in wounds with high bacterial bioburdens and stimulate healing. Journal of Plastic Surgery, 8, 151-156.

8. Ramundo, J., Gray, M., 2009: Collagenase for enzymatic debridement: a systematic review. Journal of Wound Ostomy and Continence Nursing, 36, 411.

9. Tobias, K., Johnston, S., 2012: Veterinary Surgery: Small Animal. Elsevier Saunders, Philadelphia, 2352 pp.

Received October 3, 2016

Accepted January 12, 2017 